\title{
Erratum: Search for gravitational waves from Scorpius X-1 in the second Advanced LIGO observing run with an improved hidden Markov model [Phys. Rev. D 100, 122002 (2019)]
}

B. P. Abbott, R. Abbott, T. D. Abbott, S. Abraham, F. Acernese, K. Ackley, C. Adams, R. X. Adhikari, V. B. Adya, C. Affeldt, M. Agathos, K. Agatsuma, N. Aggarwal, O. D. Aguiar, L. Aiello, A. Ain, P. Ajith, G. Allen, A. Allocca, M. A. Aloy, P. A. Altin, A. Amato, A. Ananyeva, S. B. Anderson, W. G. Anderson, S. V. Angelova, S. Antier, S. Appert, K. Arai, M. C. Araya, J. S. Areeda, M. Arène, N. Arnaud, S. Ascenzi, G. Ashton, S. M. Aston, P. Astone, F. Aubin, P. Aufmuth, K. AultONeal, C. Austin, V. Avendano, A. Avila-Alvarez, S. Babak, P. Bacon, F. Badaracco, M. K. M. Bader, S. Bae, P. T. Baker, F. Baldaccini, G. Ballardin, S. W. Ballmer, S. Banagiri, J. C. Barayoga, S. E. Barclay, B. C. Barish, D. Barker, K. Barkett, S. Barnum, F. Barone, B. Barr, L. Barsotti, M. Barsuglia, D. Barta, J. Bartlett, I. Bartos, R. Bassiri, A. Basti, M. Bawaj, J. C. Bayley, M. Bazzan, B. Bécsy, M. Bejger, I. Belahcene, A. S. Bell, D. Beniwal, B. K. Berger, G. Bergmann, S. Bernuzzi, J. J. Bero, C. P. L. Berry, D. Bersanetti, A. Bertolini, J. Betzwieser, R. Bhandare, J. Bidler, I. A. Bilenko, S. A. Bilgili, G. Billingsley, J. Birch, R. Birney, O. Birnholtz, S. Biscans, S. Biscoveanu, A. Bisht, M. Bitossi, M. A. Bizouard, J. K. Blackburn, C. D. Blair, D. G. Blair, R. M. Blair, S. Bloemen, N. Bode, M. Boer, Y. Boetzel, G. Bogaert, F. Bondu, E. Bonilla, R. Bonnand, P. Booker, B. A. Boom, C. D. Booth, R. Bork, V. Boschi, S. Bose, K. Bossie, V. Bossilkov, J. Bosveld, Y. Bouffanais, A. Bozzi, C. Bradaschia, P. R. Brady, A. Bramley, M. Branchesi,

J. E. Brau, T. Briant, J. H. Briggs, F. Brighenti, A. Brillet, M. Brinkmann, V. Brisson, P. Brockill, A. F. Brooks,

D. D. Brown, S. Brunett, A. Buikema, T. Bulik, H. J. Bulten, A. Buonanno, D. Buskulic, C. Buy, R. L. Byer, M. Cabero,

L. Cadonati, G. Cagnoli, C. Cahillane, J. Calderón Bustillo, T. A. Callister, E. Calloni, J. B. Camp, W. A. Campbell, M. Canepa, K. C. Cannon, H. Cao, J. Cao, E. Capocasa, F. Carbognani, S. Caride, M. F. Carney, G. Carullo, J. Casanueva Diaz, C. Casentini, S. Caudill, M. Cavaglià, F. Cavalier, R. Cavalieri, G. Cella, P. Cerdá-Durán, G. Cerretani, E. Cesarini, O. Chaibi, K. Chakravarti, S. J. Chamberlin, M. Chan, S. Chao, P. Charlton, E. A. Chase, E. Chassande-Mottin, D. Chatterjee, M. Chaturvedi, B. D. Cheeseboro, H. Y. Chen, X. Chen, Y. Chen, H.-P. Cheng, C. K. Cheong, H. Y. Chia, A. Chincarini, A. Chiummo, G. Cho, H. S. Cho, M. Cho, N. Christensen, Q. Chu, S. Chua, K. W. Chung, S. Chung, G. Ciani, A. A. Ciobanu, R. Ciolfi, F. Cipriano, A. Cirone, F. Clara, J. A. Clark, P. Clearwater, F. Cleva, C. Cocchieri, E. Coccia, P.-F. Cohadon, D. Cohen, R. Colgan, M. Colleoni, C. G. Collette, C. Collins, L. R. Cominsky,

M. Constancio, Jr., L. Conti, S. J. Cooper, P. Corban, T. R. Corbitt, I. Cordero-Carrión, K. R. Corley, N. Cornish, A. Corsi, S. Cortese, C. A. Costa, R. Cotesta, M. W. Coughlin, S. B. Coughlin, J.-P. Coulon, S. T. Countryman, P. Couvares, P. B. Covas, E. E. Cowan, D. M. Coward, M. J. Cowart, D. C. Coyne, R. Coyne, J. D. E. Creighton, T. D. Creighton, J. Cripe, M. Croquette, S. G. Crowder, T. J. Cullen, A. Cumming, L. Cunningham, E. Cuoco, T. Dal Canton, G. Dálya, S. L. Danilishin, S. D’Antonio, K. Danzmann, A. Dasgupta, C. F. Da Silva Costa, L. E. H. Datrier, V. Dattilo, I. Dave, M. Davier, D. Davis, E. J. Daw, D. DeBra, M. Deenadayalan, J. Degallaix, M. De Laurentis, S. Deléglise, W. Del Pozzo, L. M. DeMarchi, N. Demos, T. Dent, R. De Pietri, J. Derby, R. De Rosa, C. De Rossi, R. DeSalvo, O. de Varona, S. Dhurandhar, M. C. Díaz, T. Dietrich, L. Di Fiore, M. Di Giovanni, T. Di Girolamo, A. Di Lieto, B. Ding, S. Di Pace, I. Di Palma, F. Di Renzo, A. Dmitriev, Z. Doctor, F. Donovan, K. L. Dooley, S. Doravari, I. Dorrington, T. P. Downes, M. Drago, J. C. Driggers, Z. Du, J.-G. Ducoin, P. Dupej, S. E. Dwyer, P. J. Easter, T. B. Edo, M. C. Edwards, A. Effler,

P. Ehrens, J. Eichholz, S. S. Eikenberry, M. Eisenmann, R. A. Eisenstein, R. C. Essick, H. Estelles, D. Estevez,

Z. B. Etienne, T. Etzel, M. Evans, T. M. Evans, V. Fafone, H. Fair, S. Fairhurst, X. Fan, S. Farinon, B. Farr, W. M. Farr, E. J. Fauchon-Jones, M. Favata, M. Fays, M. Fazio, C. Fee, J. Feicht, M. M. Fejer, F. Feng, A. Fernandez-Galiana,

I. Ferrante, E. C. Ferreira, T. A. Ferreira, F. Ferrini, F. Fidecaro, I. Fiori, D. Fiorucci, M. Fishbach, R. P. Fisher, J. M. Fishner, M. Fitz-Axen, R. Flaminio, M. Fletcher, E. Flynn, H. Fong, J. A. Font, P. W. F. Forsyth, J.-D. Fournier, S. Frasca, F. Frasconi, Z. Frei, A. Freise, R. Frey, V. Frey, P. Fritschel, V. V. Frolov, P. Fulda, M. Fyffe, H. A. Gabbard, B. U. Gadre, S. M. Gaebel, J. R. Gair, L. Gammaitoni, M. R. Ganija, S. G. Gaonkar, A. Garcia, C. García-Quirós, F. Garufi,

B. Gateley, S. Gaudio, G. Gaur, V. Gayathri, G. Gemme, E. Genin, A. Gennai, D. George, J. George, L. Gergely,

V. Germain, S. Ghonge, Abhirup Ghosh, Archisman Ghosh, S. Ghosh, B. Giacomazzo, J. A. Giaime, K. D. Giardina,

A. Giazotto, * K. Gill, G. Giordano, L. Glover, P. Godwin, E. Goetz, R. Goetz, B. Goncharov, G. González,

J. M. Gonzalez Castro, A. Gopakumar, M. L. Gorodetsky, S. E. Gossan, M. Gosselin, R. Gouaty, A. Grado, C. Graef, M. Granata, A. Grant, S. Gras, P. Grassia, C. Gray, R. Gray, G. Greco, A. C. Green, R. Green, E. M. Gretarsson, P. Groot, H. Grote, S. Grunewald, P. Gruning, G. M. Guidi, H. K. Gulati, Y. Guo, A. Gupta, M. K. Gupta, E. K. Gustafson, R. Gustafson, L. Haegel, O. Halim, B. R. Hall, E. D. Hall, E. Z. Hamilton, G. Hammond, M. Haney, M. M. Hanke, J. Hanks, C. Hanna, M. D. Hannam, O. A. Hannuksela, J. Hanson, T. Hardwick, K. Haris, J. Harms, G. M. Harry, I. W. Harry, C.-J. Haster, K. Haughian, F. J. Hayes, J. Healy, A. Heidmann, M. C. Heintze, H. Heitmann, P. Hello, 
G. Hemming, M. Hendry, I. S. Heng, J. Hennig, A. W. Heptonstall, Francisco Hernandez Vivanco, M. Heurs, S. Hild, T. Hinderer, D. Hoak, S. Hochheim, D. Hofman, A. M. Holgado, N. A. Holland, K. Holt, D. E. Holz, P. Hopkins, C. Horst, J. Hough, E. J. Howell, C. G. Hoy, A. Hreibi, E. A. Huerta, D. Huet, B. Hughey, M. Hulko, S. Husa, S. H. Huttner,

T. Huynh-Dinh, B. Idzkowski, A. Iess, C. Ingram, R. Inta, G. Intini, B. Irwin, H. N. Isa, J.-M. Isac, M. Isi, B. R. Iyer,

K. Izumi, T. Jacqmin, S. J. Jadhav, K. Jani, N. N. Janthalur, P. Jaranowski, A. C. Jenkins, J. Jiang, D. S. Johnson,

A. W. Jones, D. I. Jones, R. Jones, R. J. G. Jonker, L. Ju, J. Junker, C. V. Kalaghatgi, V. Kalogera, B. Kamai,

S. Kandhasamy, G. Kang, J. B. Kanner, S. J. Kapadia, S. Karki, K. S. Karvinen, R. Kashyap, M. Kasprzack, S. Katsanevas, E. Katsavounidis, W. Katzman, S. Kaufer, K. Kawabe, N. V. Keerthana, F. Kéfélian, D. Keitel, R. Kennedy, J. S. Key, F. Y. Khalili, H. Khan, I. Khan, S. Khan, Z. Khan, E. A. Khazanov, M. Khursheed, N. Kijbunchoo, Chunglee Kim, J. C. Kim, K. Kim, W. Kim, W. S. Kim, Y.-M. Kim, C. Kimball, E. J. King, P. J. King, M. Kinley-Hanlon, R. Kirchhoff, J. S. Kissel, L. Kleybolte, J. H. Klika, S. Klimenko, T. D. Knowles, P. Koch, S. M. Koehlenbeck, G. Koekoek, S. Koley, V. Kondrashov, A. Kontos, N. Koper, M. Korobko, W. Z. Korth, I. Kowalska, D. B. Kozak, V. Kringel, N. Krishnendu, A. Królak, G. Kuehn, A. Kumar, P. Kumar, R. Kumar, S. Kumar, L. Kuo, A. Kutynia, S. Kwang, B. D. Lackey, K. H. Lai, T. L. Lam, M. Landry, B. B. Lane, R. N. Lang, J. Lange, B. Lantz, R. K. Lanza, A. Lartaux-Vollard, P. D. Lasky, M. Laxen, A. Lazzarini, C. Lazzaro, P. Leaci, S. Leavey, Y. K. Lecoeuche, C. H. Lee, H. K. Lee, H. M. Lee, H. W. Lee, J. Lee, K. Lee, J. Lehmann, A. Lenon, N. Leroy, N. Letendre, Y. Levin, J. Li, K. J. L. Li, T. G. F. Li, X. Li, F. Lin, F. Linde, S. D. Linker,

T. B. Littenberg, J. Liu, X. Liu, R. K. L. Lo, N. A. Lockerbie, L. T. London, A. Longo, M. Lorenzini, V. Loriette, M. Lormand, G. Losurdo, J. D. Lough, C. O. Lousto, G. Lovelace, M. E. Lower, H. Lück, D. Lumaca, A. P. Lundgren, R. Lynch, Y. Ma, R. Macas, S. Macfoy, M. MacInnis, D. M. Macleod, A. Macquet, F. Magaña-Sandoval,

L. Magaña Zertuche, R. M. Magee, E. Majorana, I. Maksimovic, A. Malik, N. Man, V. Mandic, V. Mangano,

G. L. Mansell, M. Manske, M. Mantovani, F. Marchesoni, F. Marion, S. Márka, Z. Márka, C. Markakis, A. S. Markosyan, A. Markowitz, E. Maros, A. Marquina, S. Marsat, F. Martelli, I. W. Martin, R. M. Martin, D. V. Martynov, K. Mason, E. Massera, A. Masserot, T. J. Massinger, M. Masso-Reid, S. Mastrogiovanni, A. Matas, F. Matichard, L. Matone, N. Mavalvala, N. Mazumder, J. J. McCann, R. McCarthy, D. E. McClelland, S. McCormick, L. McCuller, S. C. McGuire, J. McIver, D. J. McManus, T. McRae, S. T. McWilliams, D. Meacher, G. D. Meadors, M. Mehmet, A. K. Mehta, J. Meidam, A. Melatos, G. Mendell, R. A. Mercer, L. Mereni, E. L. Merilh, M. Merzougui, S. Meshkov, C. Messenger, C. Messick, R. Metzdorff, P. M. Meyers, H. Miao, C. Michel, H. Middleton, E. E. Mikhailov, L. Milano, A. L. Miller, A. Miller, M. Millhouse, J. C. Mills, M. C. Milovich-Goff, O. Minazzoli, Y. Minenkov, A. Mishkin, C. Mishra, T. Mistry, S. Mitra, V. P. Mitrofanov, G. Mitselmakher, R. Mittleman, G. Mo, D. Moffa, K. Mogushi, S. R. P. Mohapatra, M. Montani, C. J. Moore, D. Moraru, G. Moreno, S. Morisaki, B. Mours, C. M. Mow-Lowry, Arunava Mukherjee, D. Mukherjee, S. Mukherjee, N. Mukund, A. Mullavey, J. Munch, E. A. Muñiz, M. Muratore, P. G. Murray, A. Nagar, I. Nardecchia, L. Naticchioni, R. K. Nayak, J. Neilson, G. Nelemans, T. J. N. Nelson, M. Nery, A. Neunzert, K. Y. Ng, S. Ng, P. Nguyen, D. Nichols, S. Nissanke, F. Nocera, C. North, L. K. Nuttall, M. Obergaulinger, J. Oberling, B. D. O'Brien, G. D. O'Dea, G. H. Ogin, J. J. Oh, S. H. Oh, F. Ohme, H. Ohta, M. A. Okada, M. Oliver, P. Oppermann, Richard J. Oram, B. O'Reilly, R. G. Ormiston, L. F. Ortega, R. O’Shaughnessy, S. Ossokine, D. J. Ottaway, H. Overmier, B. J. Owen, A. E. Pace, G. Pagano, M. A. Page, A. Pai, S. A. Pai, J. R. Palamos, O. Palashov, C. Palomba, A. Pal-Singh, Huang-Wei Pan, B. Pang, P. T. H. Pang, C. Pankow, F. Pannarale, B. C. Pant, F. Paoletti, A. Paoli, A. Parida, W. Parker, D. Pascucci, A. Pasqualetti, R. Passaquieti, D. Passuello, M. Patil, B. Patricelli, B. L. Pearlstone, C. Pedersen, M. Pedraza, R. Pedurand, A. Pele, S. Penn, C. J. Perez, A. Perreca, H. P. Pfeiffer, M. Phelps, K. S. Phukon, O. J. Piccinni, M. Pichot, F. Piergiovanni, G. Pillant, L. Pinard, M. Pirello, M. Pitkin, R. Poggiani, D. Y. T. Pong, S. Ponrathnam, P. Popolizio, E. K. Porter, J. Powell, A. K. Prajapati, J. Prasad, K. Prasai, R. Prasanna, G. Pratten, T. Prestegard, S. Privitera, G. A. Prodi, L. G. Prokhorov, O. Puncken, M. Punturo, P. Puppo, M. Pürrer, H. Qi, V. Quetschke, P. J. Quinonez, E. A. Quintero, R. Quitzow-James, F. J. Raab, H. Radkins, N. Radulescu, P. Raffai, S. Raja, C. Rajan, B. Rajbhandari, M. Rakhmanov, K. E. Ramirez, A. Ramos-Buades, Javed Rana, K. Rao, P. Rapagnani, V. Raymond, M. Razzano, J. Read, T. Regimbau, L. Rei, S. Reid, D. H. Reitze, W. Ren, F. Ricci, C. J. Richardson, J. W. Richardson, P. M. Ricker, K. Riles, M. Rizzo, N. A. Robertson,

R. Robie, F. Robinet, A. Rocchi, L. Rolland, J. G. Rollins, V. J. Roma, M. Romanelli, R. Romano, C. L. Romel,

J. H. Romie, K. Rose, D. Rosińska, S. G. Rosofsky, M. P. Ross, S. Rowan, A. Rüdiger, P. Ruggi, G. Rutins, K. Ryan, S. Sachdev, T. Sadecki, M. Sakellariadou, L. Salconi, M. Saleem, A. Samajdar, L. Sammut, E. J. Sanchez, L. E. Sanchez, N. Sanchis-Gual, V. Sandberg, J. R. Sanders, K. A. Santiago, N. Sarin, B. Sassolas, P. R. Saulson, O. Sauter, R. L. Savage, P. Schale, M. Scheel, J. Scheuer, P. Schmidt, R. Schnabel, R. M. S. Schofield, A. Schönbeck, E. Schreiber, B. W. Schulte,

B. F. Schutz, S. G. Schwalbe, J. Scott, S. M. Scott, E. Seidel, D. Sellers, A. S. Sengupta, N. Sennett, D. Sentenac,

V. Sequino, A. Sergeev, Y. Setyawati, D. A. Shaddock, T. Shaffer, M. S. Shahriar, M. B. Shaner, L. Shao, P. Sharma, P. Shawhan, H. Shen, R. Shink, D. H. Shoemaker, D. M. Shoemaker, S. ShyamSundar, K. Siellez, M. Sieniawska, D. Sigg,

A. D. Silva, L. P. Singer, N. Singh, A. Singhal, A. M. Sintes, S. Sitmukhambetov, V. Skliris, B. J. J. Slagmolen,

T. J. Slaven-Blair, J. R. Smith, R. J. E. Smith, S. Somala, E. J. Son, B. Sorazu, F. Sorrentino, T. Souradeep, E. Sowell, 
A. P. Spencer, A. K. Srivastava, V. Srivastava, K. Staats, C. Stachie, M. Standke, D. A. Steer, M. Steinke, J. Steinlechner, S. Steinlechner, D. Steinmeyer, S. P. Stevenson, D. Stocks, R. Stone, D. J. Stops, K. A. Strain, G. Stratta, S. E. Strigin, A. Strunk, R. Sturani, A. L. Stuver, V. Sudhir, T. Z. Summerscales, L. Sun, S. Sunil, J. Suresh, P. J. Sutton, B. L. Swinkels, M. J. Szczepańczyk, M. Tacca, S. C. Tait, C. Talbot, D. Talukder, D. B. Tanner, M. Tápai, A. Taracchini, J. D. Tasson, R. Taylor, F. Thies, M. Thomas, P. Thomas, S. R. Thondapu, K. A. Thorne, E. Thrane, Shubhanshu Tiwari, Srishti Tiwari, V. Tiwari, K. Toland, M. Tonelli, Z. Tornasi, A. Torres-Forné, C. I. Torrie, D. Töyrä, F. Travasso, G. Traylor, M. C. Tringali,

A. Trovato, L. Trozzo, R. Trudeau, K. W. Tsang, M. Tse, R. Tso, L. Tsukada, D. Tsuna, D. Tuyenbayev, K. Ueno,

D. Ugolini, C. S. Unnikrishnan, A. L. Urban, S. A. Usman, H. Vahlbruch, G. Vajente, G. Valdes, N. van Bakel,

M. van Beuzekom, J. F. J. van den Brand, C. Van Den Broeck, D. C. Vander-Hyde, J. V. van Heijningen, L. van der Schaaf, A. A. van Veggel, M. Vardaro, V. Varma, S. Vass, M. Vasúth, A. Vecchio, G. Vedovato, J. Veitch, P. J. Veitch,

K. Venkateswara, G. Venugopalan, D. Verkindt, F. Vetrano, A. Viceré, A. D. Viets, D. J. Vine, J.-Y. Vinet, S. Vitale, T. Vo, H. Vocca, C. Vorvick, S. P. Vyatchanin, A. R. Wade, L. E. Wade, M. Wade, R. Walet, M. Walker, L. Wallace, S. Walsh, G. Wang, H. Wang, J. Z. Wang, W. H. Wang, Y. F. Wang, R. L. Ward, Z. A. Warden, J. Warner, M. Was, J. Watchi, B. Weaver, L.-W. Wei, M. Weinert, A. J. Weinstein, R. Weiss, F. Wellmann, L. Wen, E. K. Wessel, P. Weßels, J. W. Westhouse, K. Wette, J. T. Whelan, B. F. Whiting, C. Whittle, D. M. Wilken, D. Williams, A. R. Williamson, J. L. Willis, B. Willke, M. H. Wimmer, W. Winkler, C. C. Wipf, H. Wittel, G. Woan, J. Woehler, J. K. Wofford, J. Worden, J. L. Wright, D. S. Wu, D. M. Wysocki, L. Xiao, H. Yamamoto, C. C. Yancey, L. Yang, M. J. Yap, M. Yazback, D. W. Yeeles, Hang Yu, Haocun Yu, S. H. R. Yuen, M. Yvert, A. K. Zadrożny, M. Zanolin, T. Zelenova, J.-P. Zendri, M. Zevin, J. Zhang, L. Zhang, T. Zhang, C. Zhao, M. Zhou, Z. Zhou, X. J. Zhu, M. E. Zucker, J. Zweizig,

(The LIGO Scientific Collaboration and the Virgo Collaboration)

L. M. Dunn, S. Suvorova, R. J. Evans, and W. Moran

(Received 2 November 2021; published 23 November 2021)

DOI: $10.1103 /$ PhysRevD.104.109903

Equation (15) of the original article is in error; it should read [1]

$$
h_{0}^{\mathrm{eq}}=5.5 \times 10^{-27}\left(\frac{F_{X}}{10^{-8} \mathrm{erg} \mathrm{cm}^{-2} \mathrm{~s}^{-1}}\right)^{1 / 2}\left(\frac{r_{m}}{10 \mathrm{~km}}\right)^{1 / 4}\left(\frac{R_{\star}}{10 \mathrm{~km}}\right)^{1 / 2}\left(\frac{1.4 M_{\odot}}{M_{\star}}\right)^{1 / 4}\left(\frac{300 \mathrm{~Hz}}{f_{\star}}\right)^{1 / 2},
$$

where $r_{m}$, the accretion-torque lever arm, is evaluated at either the stellar radius $R_{\star}$ or the Alfvén radius $R_{A}$. The upper limits $h_{0}^{95 \%}$ presented in the original article are unaffected by this error.

In Fig. 5 of the original article, the theoretical torque-balance upper limit curve at the Alfvén radius should be multiplied by a factor

$$
\frac{R_{A}^{1 / 4} R_{\star}^{1 / 2}}{R_{A}^{3 / 4}}=\left(\frac{R_{\star}}{R_{A}}\right)^{1 / 2}=\left(\frac{10 \mathrm{~km}}{35 \mathrm{~km}}\right)^{1 / 2} \sim 0.534
$$

A corrected version of this figure is provided here.

In Sec. V B of the original article, the sentences

At the most sensitive sub-band, starting at $f_{0}=194.6 \mathrm{~Hz}$, the electromagnetically constrained upper limit is a factor of about 1.2 below (3.1 above) the torque balance for $R=R_{A}\left(R=R_{\star}\right)$. The upper limits for a circularly polarized signal beat the $R=R_{A}$ torque balance upper limit between 60 and $223 \mathrm{~Hz}$; and the upper limits assuming an electromagnetically constrained inclination angle beat the $R=R_{A}$ torque balance limit between $94 \mathrm{~Hz}$ and $113 \mathrm{~Hz}$.

should instead read

At the most sensitive sub-band, starting at $f_{0}=194.6 \mathrm{~Hz}$, the electromagnetically constrained upper limit is a factor of about 2.3 above (3.1 above) the torque balance for $R=R_{A}\left(R=R_{\star}\right)$. The upper limits do not beat the torque balance upper limit, either at $R=R_{A}$ or at $R=R_{\star}$.

${ }^{*}$ Deceased. 


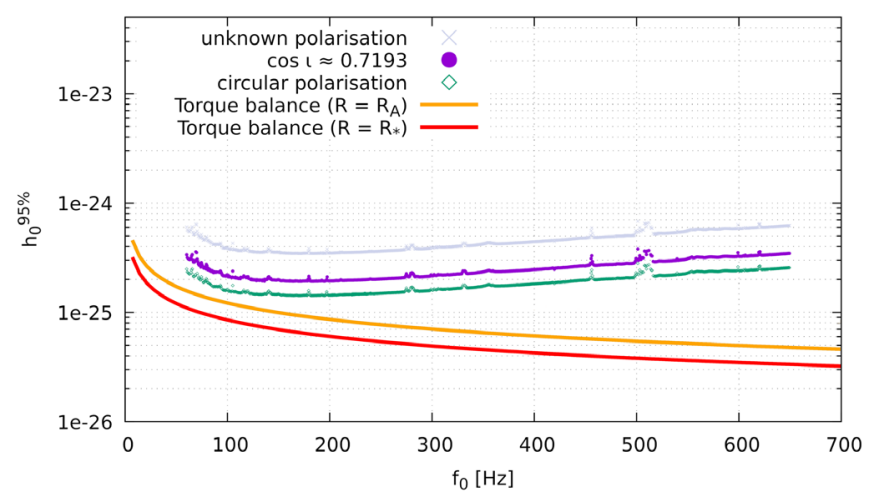

FIG. 5. Corrected version of Fig. 5 in the original article.

In Sec. VI of the original article, the sentence

For the electromagnetically restricted case, the limit is 3.1 times above, or 1.2 times below, the torque-balance limit, when the torque-balance lever arm is the stellar radius or the Alfvén radius respectively.

should instead read

For the electromagnetically restricted case, the limit is 3.1 times above, or 2.3 times above, the torque-balance limit, when the torque-balance lever arm is the stellar radius or the Alfvén radius respectively.

[1] Y. Zhang, M. A. Papa, B. Krishnan, and A. L. Watts, Astrophys. J. Lett. 906, L14 (2021). 